\title{
X-Ray Pore Optics Technologies and Their Application in Space Telescopes
}

\author{
Marcos Bavdaz, ${ }^{1}$ Max Collon, ${ }^{2}$ Marco Beijersbergen, ${ }^{2}$ Kotska Wallace, ${ }^{1}$ and Eric Wille ${ }^{1}$ \\ ${ }^{1}$ European Space Agency, Keplerlaan 1, 2200 AG Noordwijk, The Netherlands \\ ${ }^{2}$ Cosine Research B.V., Niels Bohrweg 11, 2333 CA Leiden, The Netherlands
}

Correspondence should be addressed to Marcos Bavdaz, marcos.bavdaz@esa.int

Received 12 March 2010; Accepted 25 August 2010

Academic Editor: Stephen L. O’Dell

Copyright ( 2010 Marcos Bavdaz et al. This is an open access article distributed under the Creative Commons Attribution License, which permits unrestricted use, distribution, and reproduction in any medium, provided the original work is properly cited.

\begin{abstract}
Silicon Pore Optics (SPO) is a new X-ray optics technology under development in Europe, forming the ESA baseline technology for the International X-ray Observatory candidate mission studied jointly by ESA, NASA, and JAXA. With its matrix-like structure, made of monocrystalline-bonded Silicon mirrors, it can achieve the required angular resolution and low mass density required for future large X-ray observatories. Glass-based Micro Pore Optics (MPO) achieve modest angular resolution compared to SPO, but are even lighter and have achieved sufficient maturity level to be accepted as the X-ray optic technology for instruments on board the Bepi-Colombo mission, due to visit the planet Mercury. Opportunities for technology transfer to ground-based applications include material science, security and scanning equipment, and medical diagnostics. Pore X-ray optics combine high performance with modularity and economic industrial production processes, ensuring cost effective implementation.
\end{abstract}

\section{Introduction}

With two powerful observatories already in space, X-ray astrophysics is enjoying a time of discoveries and exciting new science. XMM-Newton [1] and Chandra [2] were launched by ESA and NASA, respectively, more than a decade ago, and there is good hope that they will continue serving the science community well for another decade before consumables are exhausted or their support systems fail.

X-ray astrophysics has crucially contributed to our current understanding of the structure and history of the universe. The next generation X-ray astrophysics observatory [3] needs to employ a new X-ray optics technology, enabling a greater X-ray aperture size without sacrificing angular resolution in order to explore the Universe in even deeper detail and provide answers to the questions raised by Chandra and Newton.

High performance X-ray optics are regarded as the core enabling technology for the next-generation X-ray astrophysics observatories to follow the currently operating missions. The scientific importance of such a new space telescope is evident from the priorities expressed by the scientific communities in Europe, the USA, and Japan. In the ESA Science Programme, Cosmic Visions 2015-2025 [4, 5], the International X-ray Observatory (IXO) is one of three selected large class (L) mission candidates for a launch slot in 2020. IXO is also a high priority in the NASA and JAXA programmes and is being studied jointly by all three agencies.

European X-ray astrophysics has an impressive heritage, with a large number of missions from the early phases [6] with Exosat to the currently operating Newton [7, 8] observatory. Since such space missions have always been driving X-ray optics technology, each of them has advanced the state-of-the-art. For example, the Newton spacecraft carries three large area telescopes made from electroformed nickel shells. This technology was developed under ESA leadership in collaboration with European industry and research institutions. With this nickel optics, Newton provides a much larger collecting area (and therefore many more photons from the cosmological sources are delivered to the detector instruments) than the Chandra telescope operated by NASA (albeit at a reduced angular resolution to Chandra). It is largely due to its telescope technology that Newton is one the most productive ESA astrophysics missions to date (in terms of published papers). 
The IXO mission, a merger of the XEUS [3, 9-11] and Constellation-X [12] mission concepts, has demanding requirements on the $\mathrm{X}$-ray optics, which cannot be fulfilled with current state-of-the-art technologies. The Newton optics provide an angular resolution of about 12 arc seconds $\left({ }^{\prime \prime}\right)$ half-energy-width (HEW), while IXO requires $5^{\prime \prime}$. The effective area of the three Newton telescopes together is about $0.45 \mathrm{~m}^{2}$ at $1 \mathrm{keV}$, while IXO requires an effective area of $3 \mathrm{~m}^{2}$ to be provided, with the mass allocation to the optics only about $35 \%$ larger than that of Newton. The Chandra optics has a superb angular resolution of about $0.5^{\prime \prime}$, but a prohibitive mass when applying the same optics technology to IXO.

A number of different X-ray optics technologies have been developed to maturity, optimised, and refined for space missions, and significant investments have been made to develop each of those. Despite the fact that these missions were designed and built to achieve a range of goals, requiring diverse effective areas and angular resolutions, their performances show a clear correlation. The areal density of the optics, that is, the effective area provided by a given mass of optics, appears to be linearly dependent on the resolving power of the optics, expressed as resolution elements per angular unit; see Figure 1. The requirements for IXO, however, clearly deviate from this line; IXO needs an optics technology that can offer a much more demanding combination of mass, effective area, and angular resolution.

Additionally, the IXO aperture is large and, therefore, a timely and cost-effective production of the required optics modules must be possible.

With the invention of Silicon Pore Optics [13, 14], a solution to the challenge to meet the IXO optics technology requirements was found. The required stiffness is achieved due to the monolithic pore structure of the optic modules that are individually aligned into the optical bench, replacing the mounting approach for individual shells used in more traditional X-ray optic technologies $[12,15,16]$. The span over which mirror elements must be self-supporting is drastically reduced in the pore optic due to the rib structure between shells, as opposed to the use of a few fixed mounting points in the traditional approach. This allows the mirror substrate thickness to be drastically reduced, allowing a much denser packing of mirror shells, thereby increasing the effective aperture without compromising the optics stiffness and figure accuracy. Compared to the replicated nickel shell $\mathrm{X}$-ray optics technology the mass is reduced by an order of magnitude.

\section{Silicon Pore Optics (SPO)}

2.1. A Novel Approach to Making X-Ray Optics. The mounting concepts of the X-ray optics technologies used by the missions flown to date and plotted in Figure 1 share one common aspect. The optical mirror elements, be it closed shells or shell segments, are attached to the support structure on individual points. In most cases, a spider structure is used, to which the optical mirror element is attached at the intersection points. These mounting interfaces are rather

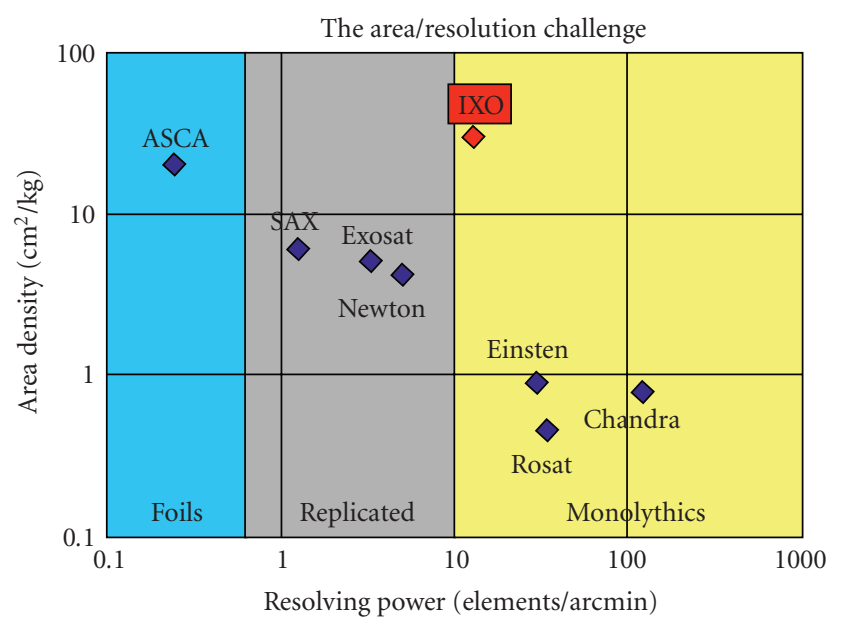

Figure 1: The effective area versus angular resolution challenge: A clear correlation is noted, when plotting the mass required to provide a given effective area as a function of the angular resolving power, expressed as resolution elements per angular unit. The X-ray optics flown to date can be separated into three groups: foil based optics, replicated shells and polished monolithic optics. Although these are very different from each other, the same area/resolution correlation is valid for all of them. The optics for each of the flown missions has been carefully optimised and raised to a high level of maturity. IXO requires a truly novel optics technology, clearly off the correlation line. The IXO optics technology has to be able to provide much more effective area per given mass, whilst maintaining the angular resolution performance than any of the preceding missions to date.

localised and involve mechanical clamping or glue-spots to form the connection. In Figure 2, images $\mathrm{A}$ and $\mathrm{B}$, the mounting concept for closed shells and sectors, respectively, is sketched.

The Silicon Pore Optics relies on a different mounting concept $[13,14,17,18]$. The X-ray mirror elements are mounted along densely spaced lines, via ribs, which attach to the back of the mirror element; see Figure 2, image C. These mounting elements (ribs) have two functions: (1) they provide stiffness to the mirror element in the decisive longitudinal direction (parallel to the optical axis), and (2) they distribute the load over a line and not a point. The mirror element becomes much stiffer, and its figure much less distorted by the mounting elements.

The pore structure is obtained when mirror elements are stacked, attached to each other front-to-back, as illustrated in Figure 2, image D. The ribs on adjacent mirror elements are coaligned, jointly generating very strong structural walls. These radial walls, each of their extensions containing the optical axis, provide strength in the direction normal to the optical surface. Due to their dense packing, the required thickness of these walls is very small and the overall loss in geometric area is, therefore, comparable with that of the classical spider obscuration.

In the SPO technology, the mounting ribs are made of the same material as the mirror elements. Actually, the mirror element and the ribs are manufactured from a single 


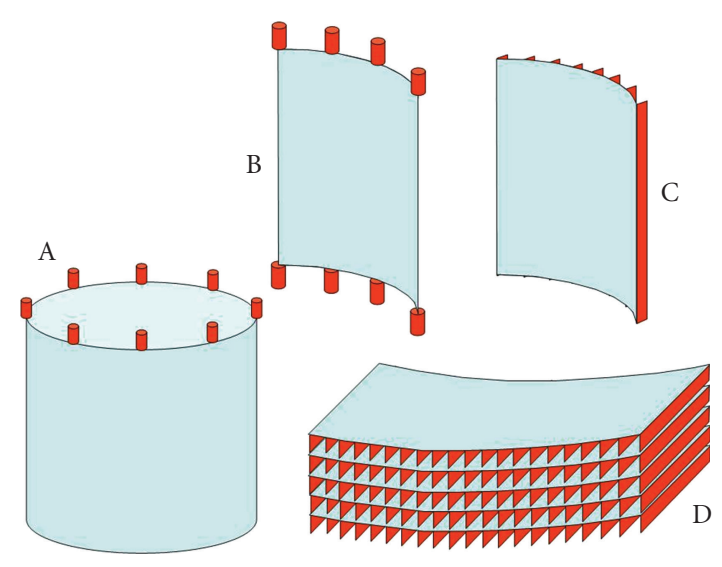

FIGURE 2: The mounting concept of traditional X-ray optics technologies is illustrated in images $\mathrm{A}$ and $\mathrm{B}$. The closed mirror shells or sectors are mounted to the support structure at individual points. In the Silicon Pore Optics technology ribs attached to the back of the mirror elements form the mounting elements (image C). These ribs dramatically increase the stiffness of the mirror elements in the critical longitudinal direction. Mirror elements are stacked upon each other in the SPO approach, forming a stiff, monolithic structure containing many mounted mirror elements (image D).

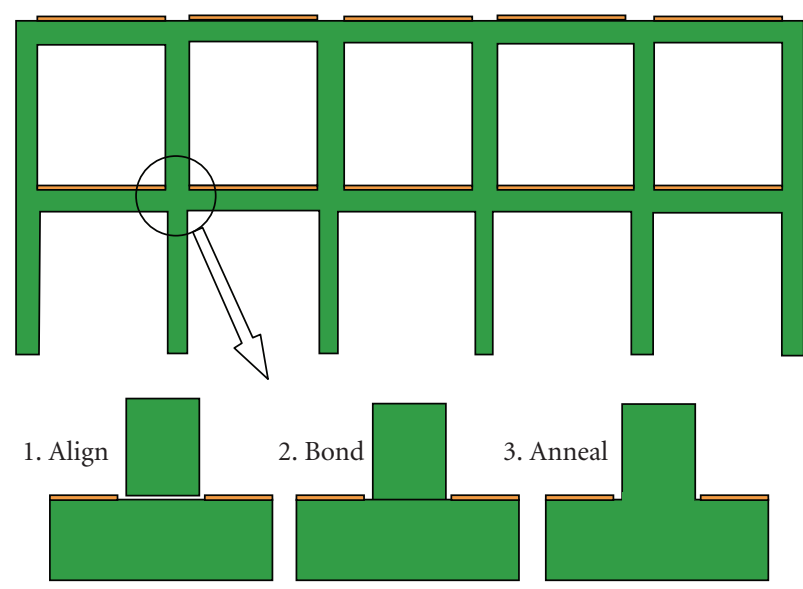

Figure 3: The mirror elements are joined using optical bonding, avoiding the use of glue or cement with its associated shrinkage and CTE mismatch problems. The mirror element stack is, therefore, a monolithic silicon crystal. If required, the mirror elements can be coated before the stacking process.

piece of silicon crystal. Therefore, the thermal expansion coefficient (CTE) of the mirror elements and ribs is identical. Individual, ribbed mirror elements are attached to each other using optical bonding. The surface of the rib tops, as well as the optical surfaces, must be of sufficient quality to ensure that the surfaces of adjacent plates bond to form a single monolithic structure. No glue is required, which would cause shrinkage and CTE mismatch problems. The stacking process and the merging of adjacent optical mirror elements is shown schematically in Figure 3. The mirror elements are first accurately aligned, then contacted to form a bond, and finally annealed to improve the strength of the joint. In Figure 3 , it is also indicated by the orange lines, that the mirror elements can be coated as required before the stacking process, in order to increase the X-ray reflectivity. Note also that the unavoidable residual stress introduced by the coating (with its different CTE) is easily handled by the stiffness of the mirror element stack.

Considering the large number of required optics modules for a mission like IXO, it was very important to take into account mass production aspects right from the beginning of the technology development. Due to the small size of the SPO modules the production equipment can be kept compact, ensuring the cost-effective implementation of a production line, including the associated cleanroom infrastructure.

The production of the telescope optics for a large mission like IXO can be split into two streams, as indicated in Figure 4: (1) the production of SPO modules and (2) the integration and assembly of the SPO modules into the complete telescope. These two streams can be established at different geographic locations and assigned to different management structures if required.

2.2. Production of Silicon Pore Optics. X-ray optics require superpolished mirror surfaces. Similar requirements are imposed on the surface finish of the latest generation silicon wafers for the electronics industry. These are already commercially mass produced and very substantial investments have been made by the semiconductor industry to achieve the high quality surface finish. The surface roughness corresponds to that required for X-ray optics and the figure errors are within the error budget for a few arc second angular resolution optic $[19,20]$. In addition, the surfaces of such double-sided polished wafers are very parallel, with very small thickness variations.

As illustrated in Figure 5, the starting material is procured and then processed for use in an SPO, relying largely on existing and modified processes available in the semiconductor industry. New processes and associated tools are developed only where required. The silicon pore optics production can be grouped as follows:

(a) production of ribbed and wedged silicon plates,

(b) coating of the plates,

(c) assembly into stacks of tens of plates,

(d) assembly of two stacks to form a mirror module.

The production of silicon mirror plates [21] starts with dicing SEMI standard $\langle 100\rangle 300 \mathrm{~mm}$ silicon wafers, which are double sided polished and $0.775 \mathrm{~mm}$ thick, into square plates. The diced plates are then coated with a protective layer to prevent damage to the superpolished surface during the subsequent production steps (see Figure 6). The ribbing process dices grooves into the silicon substrate, thereby creating

(i) the pores forming the channels for the $\mathrm{X}$-rays to pass through,

(ii) the ribs providing the required structural stiffness when bonded in a stack, and

(iii) a thin membrane. 

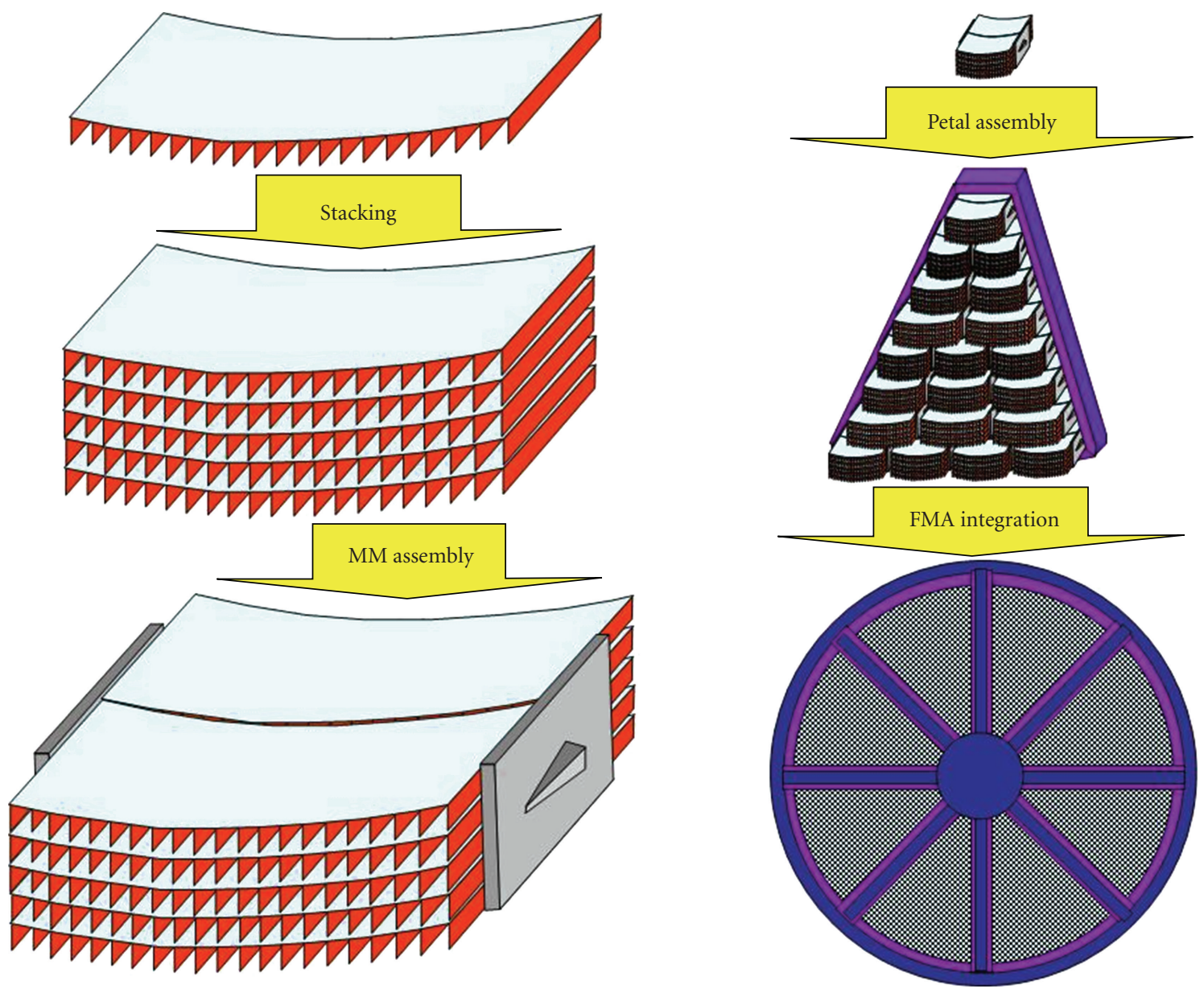

FIgURE 4: The production streams for a telescope using SPO technology.

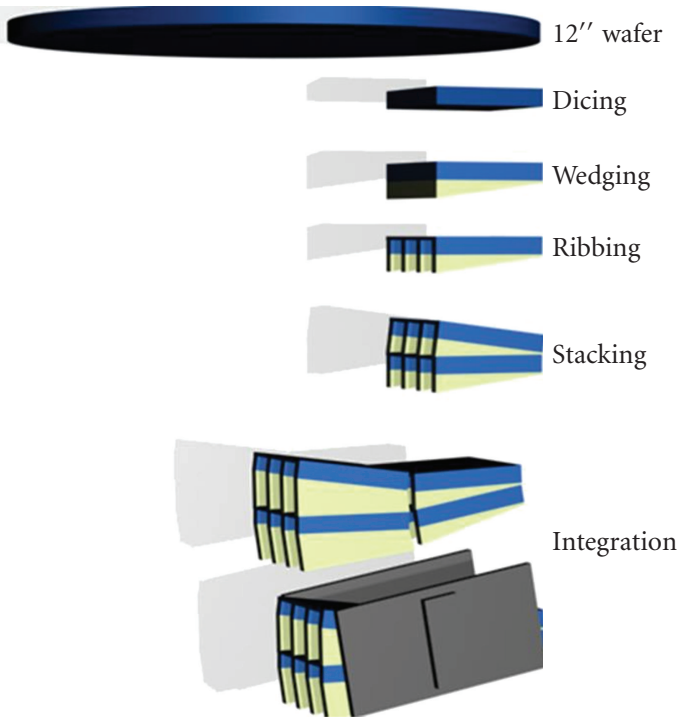

FIGURE 5: The production of SPO modules starts with commercial silicon wafers and utilises, as far as possible, existing methods and processes. The stacking is done automatically using a stacking robot.
Parameters such as rib width, pitch, number of ribs, and membrane thickness can be optimised for the specific application. Typically the ribs are $0.17 \mathrm{~mm}$ wide with a $1 \mathrm{~mm}$ pitch and a membrane thickness of $0.17 \mathrm{~mm}$. A slightly modified standard semiconductor dicing saw is used for cutting the channels.

During stacking the plates will be elastically deformed [22] to create approximations of the curved surfaces of a Wolter-I optic [23]. To minimise the strain energy, one reduces the membrane thickness to a level sufficient to meet the figure requirements of the final optic. Although the plate ribbing process results in a well-defined pore geometry, a known side effect of dicing is the generation of microcracks in the material. Therefore, a second step in the shaping of the required rib geometry has to be applied to remove any residual microcracks, a so-called damage etch. A potassium hydroxide $(\mathrm{KOH})$ solution is used to anisotropically and selectively etch damaged silicon material inside the diced grooves, while a protective coating is preventing the top surface of the ribs from being etched (see also Figure 6). Although the surface of the diced grooves remains rough after damage etching, this will not affect the optical performance of the telescope. In contrast, surface 


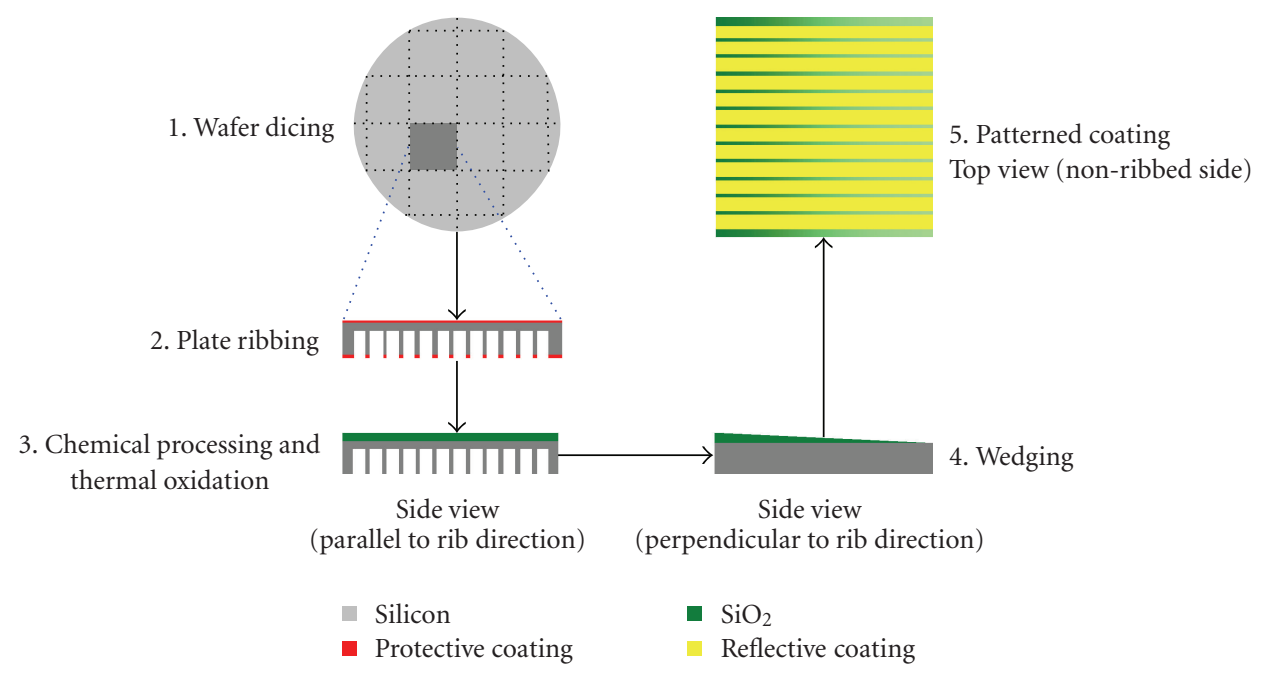

FIGURE 6: Schematic overview of process flow for production of Si mirror plates (Reproduced from [20]).

roughness within the grooves helps to absorb or at least scatter unwanted X-rays. Since in the SPO manufacturing one has full access to the pores during manufacturing of the plates, one could also apply other surface roughening techniques to further reduce stray light.

The goal of the wedging process is to taper plates along the optical axis so as to create, when they are elastically bent and stacked, a conical approximation to a Wolter I Optic (Figure 7). The wedge angle is proportional to the pore height, inverse proportional to the focal length of the optic and, therefore, independent from the radial position of the plate. Typically the wedge angle is a few $10 \mu \mathrm{rad}$, resulting on plate lengths of $66 \mathrm{~mm}$ in a wedge layer with a maximum thickness of a few $100 \mathrm{~nm}$. The tapered wedge of the plates is applied by controlled etching of the plates in an isotropic etchant solution using custom-built equipment. The wedge angle accuracy directly influences the optical performance and has, therefore, to be controlled to 10 microarcsecond level. Dedicated equipment has been developed and reliably produces wedges well within this tight tolerance.

After wedging, a final processing step is necessary for optimization of the reflectivity of the mirror plates. High- $Z$ metallic coatings such as platinum, tungsten, gold, or iridium are typically used to increase the reflectivity [24]. Patterning of the coated surface by metallic masks [25] or lithographic processes [21] is necessary to ensure bondability of the silicon mirror plates during the stacking process.

The silicon plate surfaces can be bonded either by hydrophilic or by hydrophobic bonding. Hydrophobic bonding occurs between two silicon layers which are typically made by removing the native oxide. Hydrophilic bonding occurs between two oxide layers, which can be native oxide layers of a few angstroms in thickness or thermal oxide layers of hundreds of nanometers. The bond strength of room temperature direct bonded wafers is highest for native oxide $\left(83 \mathrm{~mJ} / \mathrm{cm}^{2}\right)$ and thermal oxide $\left(52 \mathrm{~mJ} / \mathrm{cm}^{2}\right)$ and lowest for hydrophobic Si $\left(10-20 \mathrm{~mJ} / \mathrm{cm}^{2}\right)$ [26]. Note that the bond strength can be increased by a factor 10-20 if the bonded stacks are annealed.

The wedged mirror plates are then cleaned and elastically bent, using a fully automated stacking robot, into a conical shape. A flexible die is used to set the appropriate radius of curvature. The Wolter-I geometry of a parabolic and a hyperbolic mirror can, for long focal lengths, be approximated by two cones ("conical approximation"). When stacked the mirrors remain flat along the pores. The achievable angular resolution is then limited by the height of a single pore [27]. In the case of IXO with a focal length of $20 \mathrm{~m}$ and using pores with a height of $0.6 \mathrm{~mm}$, this results in a lower limit to the half-energy width (HEW) of about $3^{\prime \prime}$. To reduce the lower limit, we explore shaping the mirrors also in longitudinal direction.

The fully automated assembly robot (Figure 8 ) is specifically developed $[28,29]$ to stack silicon pore optics and is a combination of standard semiconductor systems and newly developed tools. The complete system has a footprint of a few $\mathrm{m}^{2}$ only and is installed in a class 100 clean area. The robot selects a plate for stacking and inspects it for particles. The plate is then handed over to the actual stacking tool, which will elastically bend it into a cylindrical or conical shape. This tool, called a die, is then lowered onto the mandrel, where it will deposit the plate, or stack it, onto already existing ones. The die and the mandrel are supervised by metrology systems based on autocollimators, cameras and force sensors. Note that only the figure of the mandrel is replicated, not its roughness. The stacking is done from outer radii inwards, thus always exposing the last integrated mirror surface to the metrology tools.

Multiple mirrors stacked on top of each other form together a stack, in which the X-rays are reflected off the reflective membrane inside each pore. Due to the inherent stiffness of the stacks, the figure of the individual mirrors remains preserved during further mounting and integration. Two of such stacks are coaligned and integrated into brackets to form a so-called mirror module. 


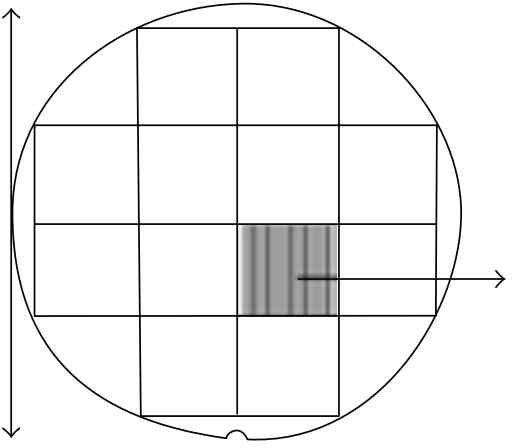

(a)

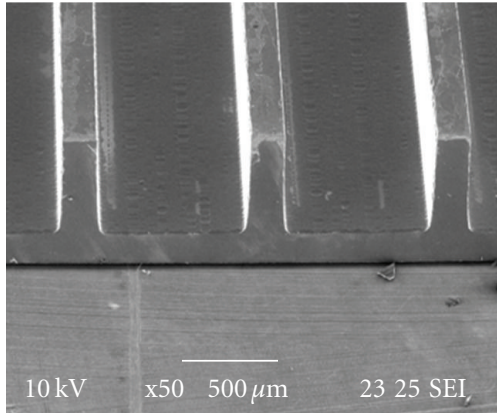

(b)

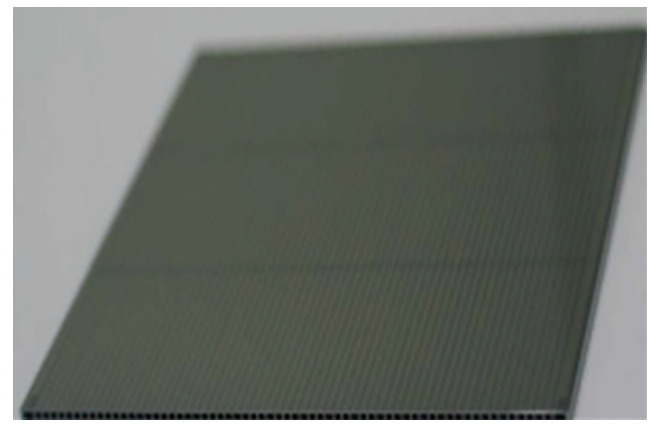

(c)

Figure 7: (a) Silicon Pore Optics are made from commercial high-quality $12^{\prime \prime}$ silicon wafers which are diced into plates. (b) The plates are ribbed (reflecting surface pointing downwards). The $0.17 \mathrm{~mm}$ wide ribs have a pitch of $1 \mathrm{~mm}$ and the membrane is $0.17 \mathrm{~mm}$ thick. The plates are then wedged along the rib direction (not shown) and a patterned iridium coating is applied on the reflective surface (c). The pattern keeps the areas free where the next plate will be bonded. The plate shown has dimensions of $66 \times 66 \mathrm{~mm}^{2}$ and a thickness of $0.775 \mathrm{~mm}$. Photographs courtesy of Micronit (b) and DNSC (c).

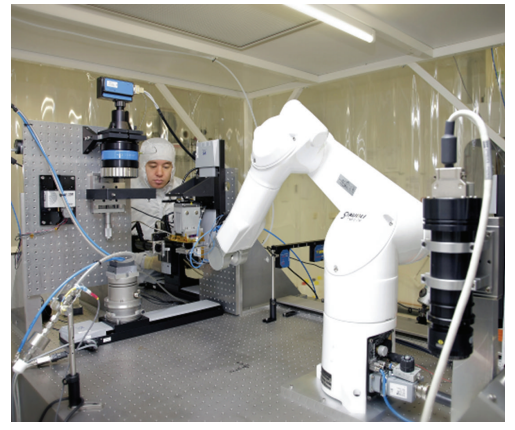

(a)

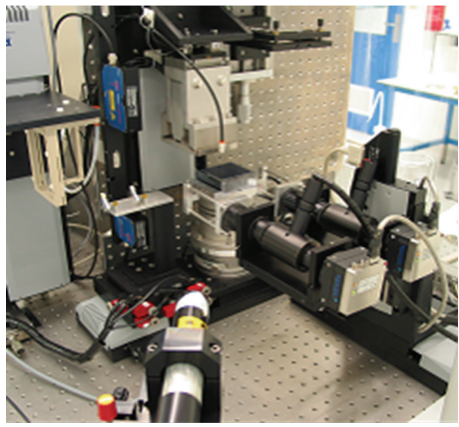

(b)

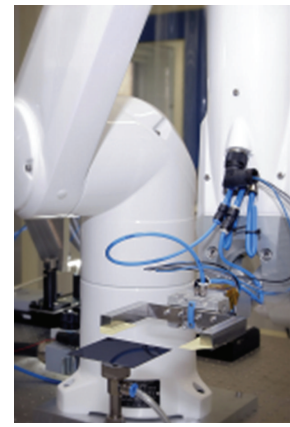

(c)

FIGURE 8: Stacking robot inside the class 100 clean area at cosine. The system is installed on a vibration isolated table, consists of more than 16 axes, is fully automated, and is designed to build stacks up to 100 plates high. The plates can be positioned with $\mu \mathrm{m}$ accuracy and automatically be bent into the required shape. Photographs courtesy of cosine Research BV.

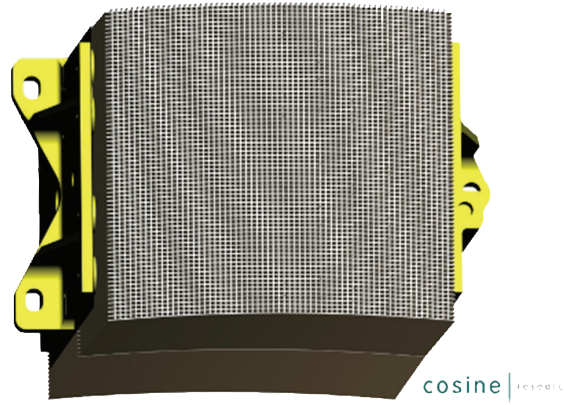

(a)
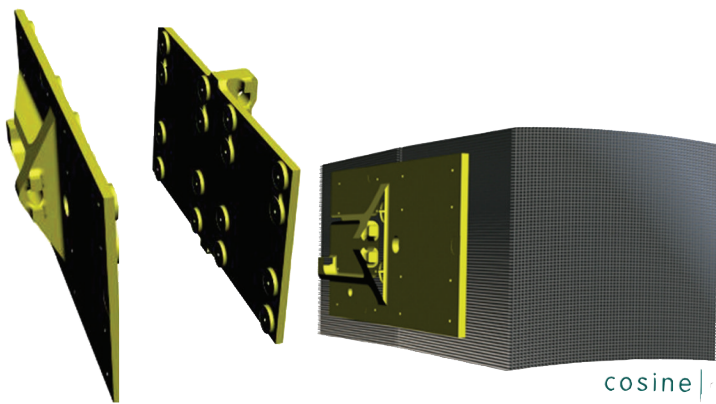

(b)

Figure 9: A mirror module, consisting of two mirror stacks, coaligned to form a Wolter-I optics, is fixed by two CeSiC brackets. These brackets provide glue pads, petal interfaces, and integration interfaces. The area covered by the bracket is $92 \times 47 \mathrm{~mm}^{2}$ and both brackets together have a weight of $56 \mathrm{~g}$.

Several concepts on how to fix two stacks together, and thereby maintain particularly the tight tolerance on the kinkangle required for the Wolter 1 configuration, have been evaluated and traded [19]. The outcome was a simple design (Figure 9) consisting of two brackets, which are glued onto the two stacks, once these are coaligned. This very lightweight solution uses the intrinsic stiffness of a pore structure to form a rigid X-ray lens. The brackets have in total three interface points to the optical bench, which allow for radial translation and rotation around the optical axis. The bracket material of 


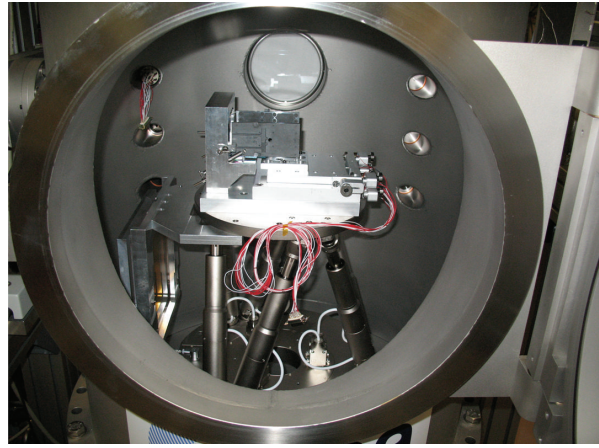

(a)

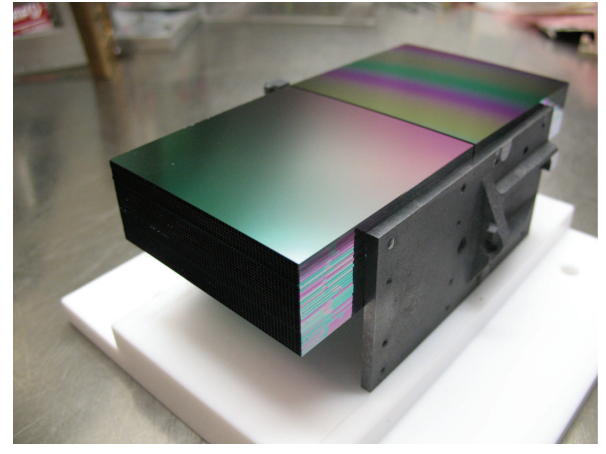

(b)

Figure 10: (a) A mirror module inside the vacuum tank of the PTB X-ray test facility at the BESSY synchrotron in Berlin. (b) A mirror module consisting of two wedged stacks coaligned to arc-second level and fixated together by two silicon carbide mounting brackets. Photographs courtesy of ESA/PTB (a) and cosine Research BV (b).

choice is $\mathrm{CeSiC}$, a silicon carbite ceramics, because it matches the CTE of silicon, has a high thermal conductivity, can be easily machined, and has excellent mechanical properties.

Several integration concepts were analysed in order to achieve the challenging requirement of $1^{\prime \prime}$ for coalignment of two stacks. The simplest solution found was to align the optics under active X-ray illumination, since it simultaneously tests the optics at the wavelength under which it will operate and allows to determine the optical axis for integration into the optical bench $[29,30]$. For this purpose, full beam illumination can be used; however, the required integration times can be quite long and an image is obtained that convolves alignment errors and possible figure errors. Pencil beam testing using a synchrotron source has been already successfully used to characterise silicon pore optics and has also been found to be well suited for measuring the coalignment of a mirror module. It yields a higher spatial resolution and the integration time on the CCD is only a few seconds (Figure 10).

A dedicated beamline has been set up in the laboratory of the Physikalisch-Technische Bundesanstalt (PTB) at the synchrotron radiation facility of BESSY [31]. A $2.8 \mathrm{keV}$ pencil beam of dimension ranging from $1 \mathrm{~mm}$ down to $50 \mu \mathrm{m}$ is used to scan the sample. After double reflection of the beam an image is recorded by the CCD, which is located at $5 \mathrm{~m}$ distance from the sample, providing arcsecond pixel resolution. The beam is used to probe the sample and the information thereby obtained is correlated with results obtained from interferometry during assembly of the stacks. Since the mirror module is then essentially an $\mathrm{X}$-ray lenslet, residual integration errors can be partially corrected during integration into the optical bench [32] (see Figure 11), namely, by radial translation and rotation around the optical axis.

Currently, conical approximations to the Wolter 1 geometry are the baseline for the technology development, since metrology and the associated data analysis is somewhat simplified. In the case of IXO, the conical approximation contributes about $3^{\prime \prime}$ to the HEW budget. Once the angular resolution performance of the SPO modules produced will

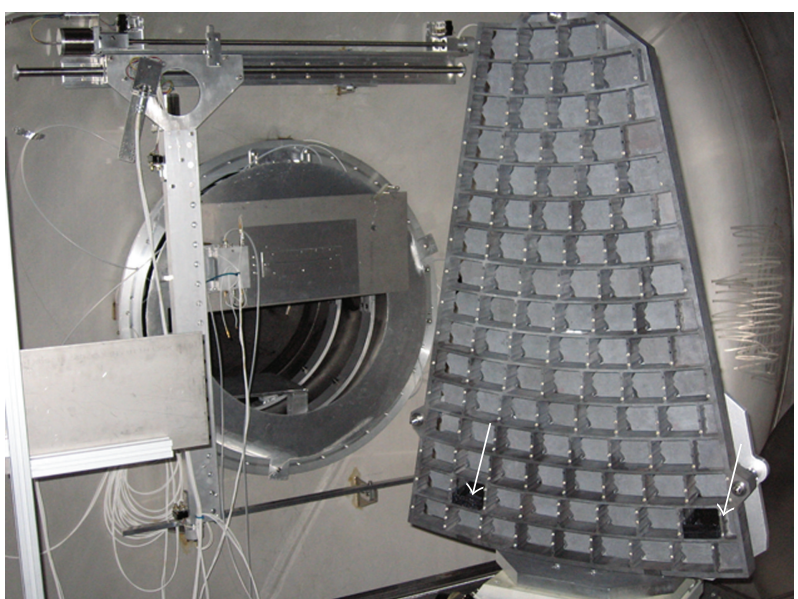

FIGURE 11: Cesic optical bench ("petal”) with a height of $1.1 \mathrm{~m}$ inside the PANTER X-ray test facility, with two silicon pore optics mirror modules mounted (marked by white arrows) in flight representative configuration. Photograph courtesy of MPE/KayserThrede.

approach closely the IXO requirements, the technology developments will start to use true Wolter geometries. The implication on the SPO production of moving to true Wolter 1 optics are fully understood, and the required equipment modifications have already been considered. Preliminary tests have demonstrated the required elastic bending of the mirror plates.

2.3. Performance of Silicon Pore Optics. The quality of silicon pore optics is measured throughout the production process [19]. After the plate has been stacked, its figure is measured using an interferometer equipped with a computer-generated hologram acting as nulling lens. Figure errors can be measured to $\lambda / 20$, and the surface deviation measurements indicate whether residual particles have been trapped and what size they had. From these interferograms, it is possible to predict the $\mathrm{X}$-ray performance of the mirrors. 
XOU-3: for comparison: plates 1, 4, 8 (EPIC-pn) XOU-5: improvement: plates 1, 4, 8 (PSPC)
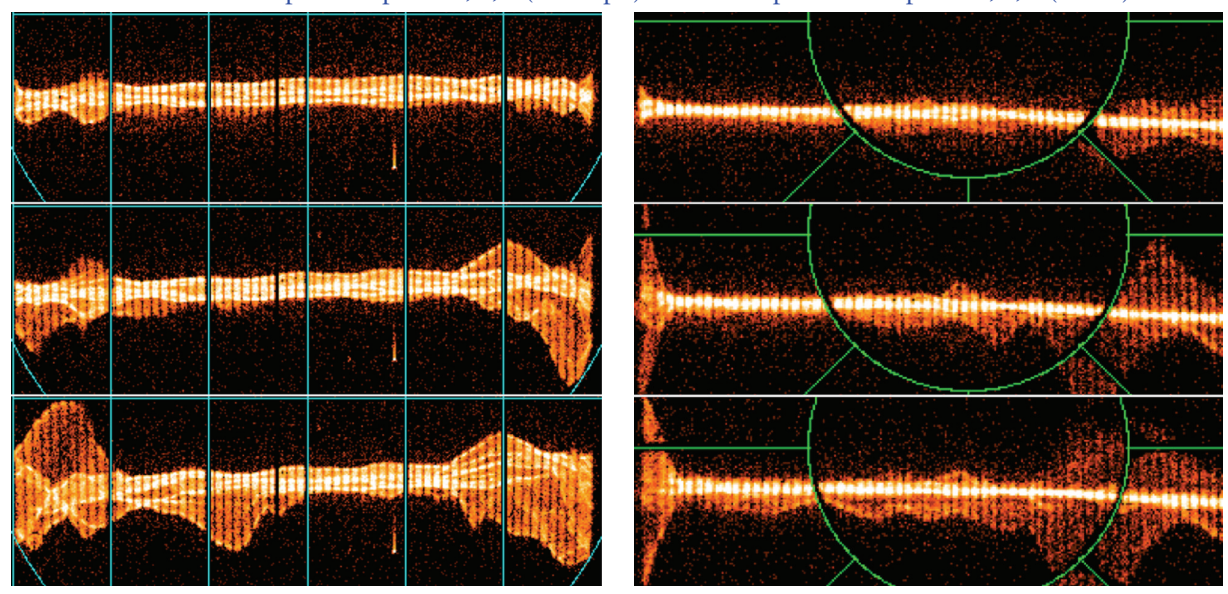

FIGURE 12: Examples of intrafocal measurements (camera was positioned 8 meters behind the optics) of the two mirror modules at the PANTER facility. The mirror module on the left was produced in 2007, the one in the right in 2009. From top to bottom plates 1 , 4 , and 8 are shown. The measurements have been taken at an energy of $3 \mathrm{keV}$. Such measurements allow to estimate the alignment of the individual mirror plates and to identify the location and magnitude of figure errors.

Full area illumination is used to characterise the optics, to measure scattering off the ribs, and to perform reflectivity as function of energy measurements.

Figure 12 shows a comparison of two different mirror modules, assembled between 2007 and 2009, and measured using $3 \mathrm{keV}$ X-rays at the PANTER facility [33] of the Max Planck Institute für extraterrestrische Forschung (MPE). The figure shows a comparison of plates 1,4 , and 8 . The significant improvement in quality is clearly visible from the direct comparison of the double reflected image measured under an incidence angle of $0.5 \mathrm{deg}$ at a distance of $8 \mathrm{~m}$.

Pencil beam X-ray measurements, measured at PTB, are used during alignment and integration of a mirror module and are then used to predict its optical performance by reflecting a small X-ray beam at grazing incidence of the surface of every plate/pore. These measurements allow determining the angular deviation of the mirror surface in each pore of a mirror module. Using raytracing algorithms [34], this allows us then to predict the PSF and HEW of the optic in the focal plane. In combination with the data taken from the individual stacks (see Figure 13), this gives additional information on how stack up errors propagate.

In November 2009, a mirror module (XOU5) was then measured in double reflection, mounted in flight representative configuration (i.e., the mirror module was mounted in a petal structure as required for IXO). From the results measured at $5 \mathrm{~m}$, one can extrapolate an estimate for the PSF expected in the focal plane, shown in Figure 14 for the first 4 and for 20 plates.

Figure 15 shows the measured performance of the first assembled mirror modules made from silicon pore optics and an estimate for the further evolution. The quality of the optics has drastically improved with increased cleanliness of all involved process steps. The technological issues of cleanliness and bonding are being addressed by improved assembly hardware, developed in the course of the ongoing technology research program and during the steep learning curve in assembling pore optics. It shall be noted that so far no show stopper has been identified that would impede improving the performance of silicon pore optics beyond $5^{\prime \prime}$.

2.4. International X-Ray Observatory. Silicon Pore Optics technology enables future X-ray telescopes with a large effective area and high angular resolution. The International $\mathrm{X}$-ray Observatory (IXO) is an L-class mission candidate within ESA's Cosmic Vision 2015-2025 programme [5]. IXO is developed with the joint efforts of NASA, ESA, and JAXA and launch is planned for around 2021.

The key requirements of the IXO X-ray mirror are an effective area of $3 \mathrm{~m}^{2}$ at $1.25 \mathrm{keV}$ and an angular resolution of 5 arcsec HEW. Multilayer coatings on the inner part of the mirror will provide enhanced reflectivity for the 10 $40 \mathrm{keV}$ range, with an effective area of $150 \mathrm{~cm}^{2}$ at $30 \mathrm{keV}$ and 30 arcsec angular resolution. The available launchers limit the mass of the mirror assembly to $2000 \mathrm{~kg}$, including mounting structure and thermal control. This requires a mirror technology with a very high area-to-mass ratio of $20 \mathrm{~cm}^{2} / \mathrm{kg}$, being 50 (8) times larger than for Chandra [2] (XMM Newton [1]). Two different approaches are studied by IXO: Silicon Pore Optics by ESA and Segmented Glass Optics by NASA.

The mirror diameter is $4 \mathrm{~m}$, limited by the size of the fairing. To allow small grazing angles of incidence, a large focal length between $20-25 \mathrm{~m}$ is required. This is realized by connecting the mirror and the instrument modules by a deployable structure (see Figure 16). The spacecraft is folded to fit into the launcher fairing and deployed once in space.

IXO will contain five different instruments for imaging, spectroscopy, polarimetry, and timing measurements. 


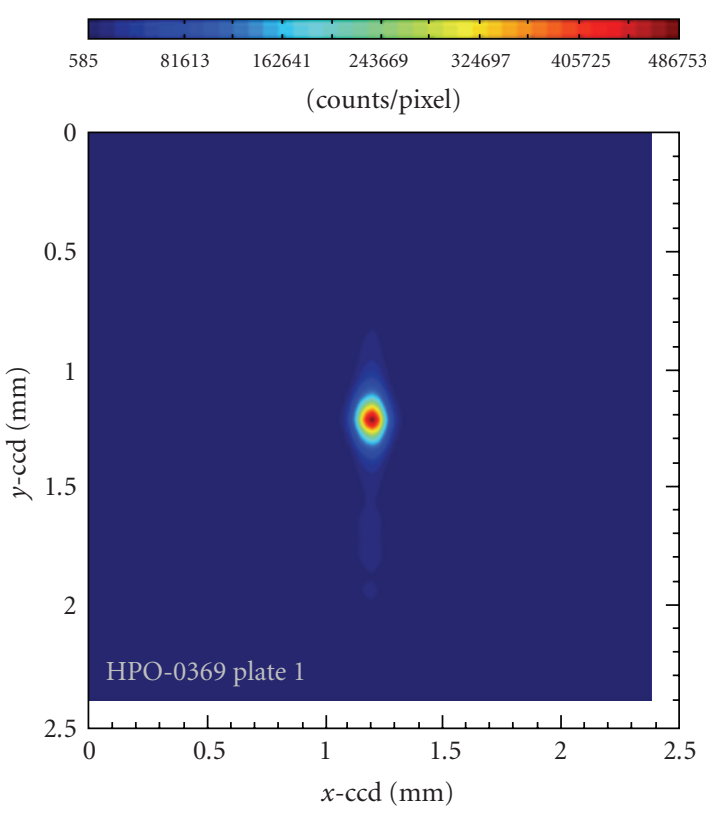

(a)

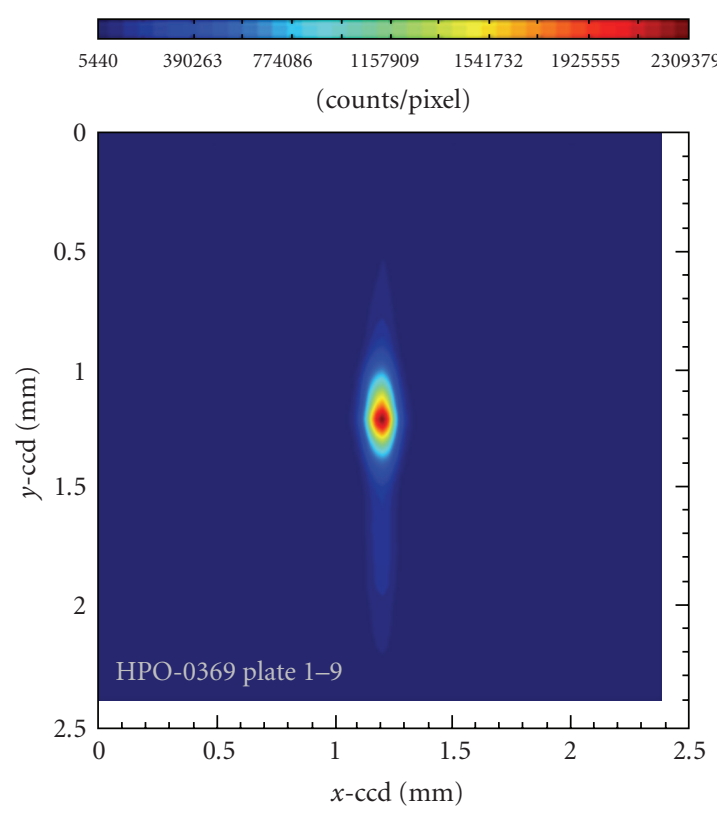

(b)

FIGURE 13: (a) PSF of the first plate (bottom) of a stack of 9 wedged plates measured using established procedures in single reflection at $2.8 \mathrm{keV}$ at the FEM beamline in the PTB lab at the BESSY synchrotron radiation facility. The full length of the plate was scanned using a $100 \mu \mathrm{m}$ pencil beam with an intrinsic HEW of $4^{\prime \prime}$. The scans where repeated every $2 \mathrm{~mm}$ over the full width of the plate. The resulting PSF, excluding the direct beam, has a HEW of 4.2". (b) The same measurement repeated on the entire stack of 9 plates. The HEW of the PSF, excluding the direct beam, is $7^{\prime \prime}$. In double reflection this would result in a HEW of a mirror module of $10^{\prime \prime}$.

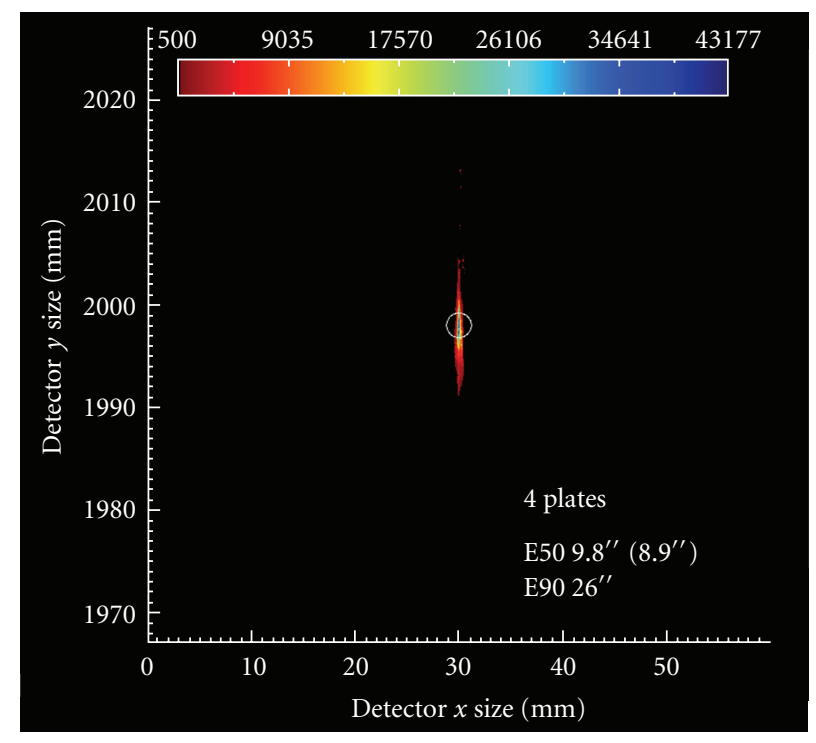

(a)

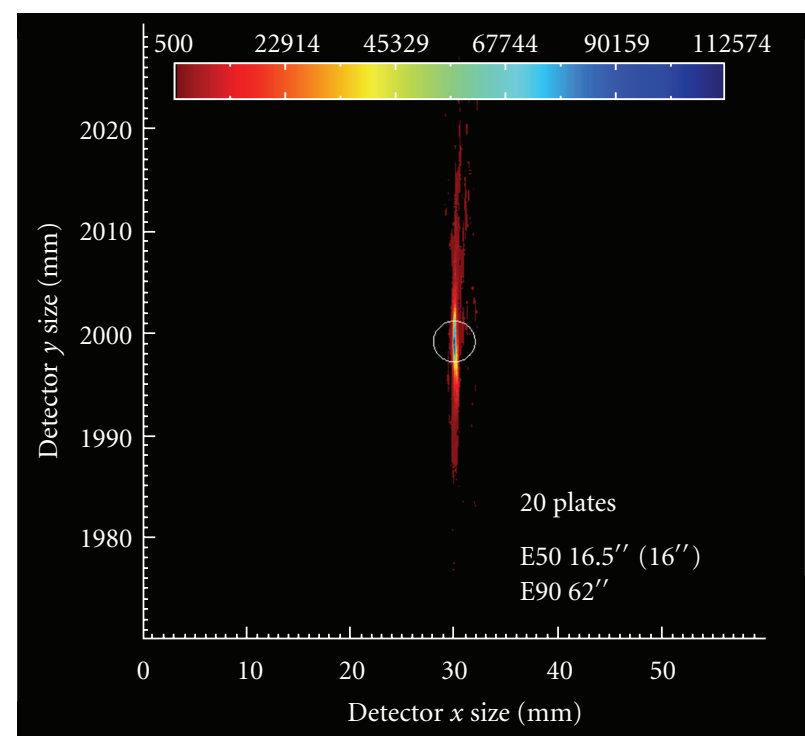

(b)

FIGURE 14: PSF of the first 4 (a) and 20 (b) plates of a mirror module consisting of 20 wedged plates measured using established procedures in double reflection at $2.8 \mathrm{keV}$ at the FEM beamline in the PTB lab at the BESSY synchrotron radiation facility. The full length of the plate was scanned using a $100 \mu \mathrm{m}$ pencil beam with an intrinsic HEW of $4^{\prime \prime}$. The scans where repeated every 2 mm over the full width of the plate. The resulting PSF, excluding the direct beam, has a HEW of $8.9^{\prime \prime}$ (4 plates) and 16" (20 plates).

Their details can be found in $[35,36]$. The large effective area of the telescope will enable observations that are not possible with Chandra or XMM Newton, but are crucial for a quantitative understanding of the X-ray universe.

\section{Glass Micropore Optics (MPO)}

This optic type is less performing in terms of angular resolution but extremely efficient with regard to the mass allocation, making it of particular interest for applications on 


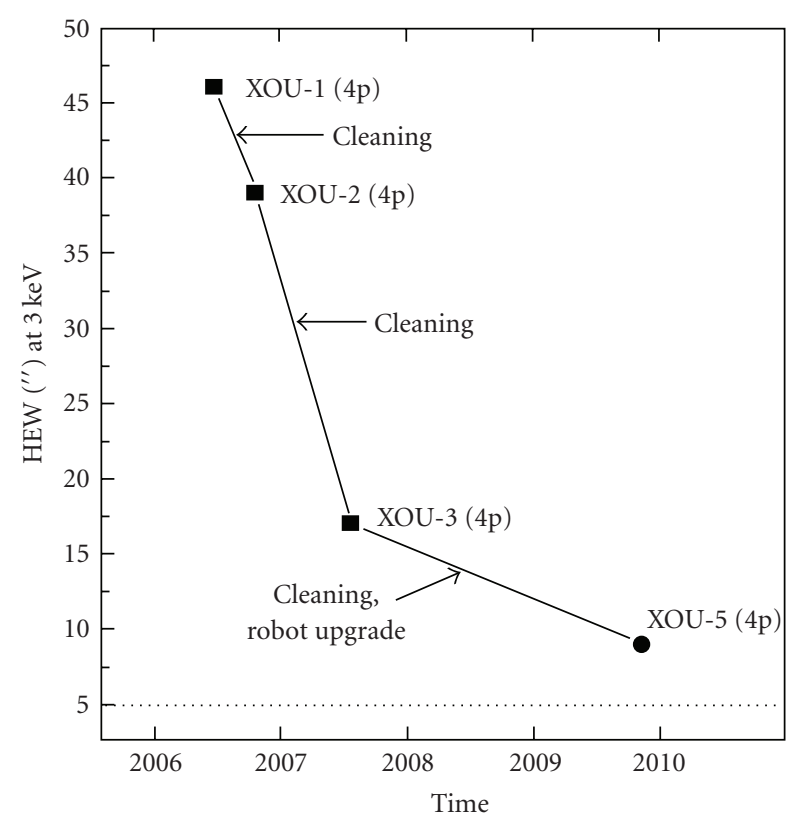

FIGURE 15: Performance of mirror modules of silicon pore optics measured (solid blocks) in mounted configuration, measured with $\mathrm{X}$-rays at $3 \mathrm{keV}$.

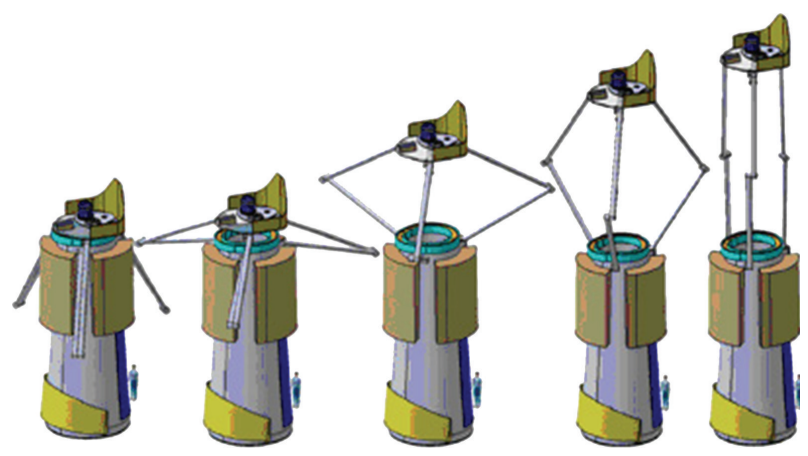

FIGURE 16: Schematic diagram of IXO showing the articulated arm deployment system (shroud not shown) [35].

planetary missions. Collimating elements still form the backbone of X-ray instrumentation for planetary exploration and science. With the development of coated, square pore micropore optics for X-ray imaging optics, this situation changed.

3.1. Building on Night Vision. Glass micropore optics (as opposed to silicon micropore optics [37], which is produced by micromachining silicon) are based on the Microchannel Plate (MCP) technology that has been developed for many decades for image intensifiers and photon and particle detectors. One method to produce microchannel plates is to cut thin slices off fused bundles of thin round glass fibres and etch out the fibre cores. The glass fibres have a cladding of a different type of glass than the core, and the fibre bundle is fused at a temperature where the cladding glass melts together but the core glass is unmodified. The slices are etched such that the core glass of all fibres is etched away, leaving behind the cladding glass. The result is a glass plate, of about 0.5 to a few $\mathrm{mm}$ thick, with a high density of cylindrical holes with diameters between 3 and $100 \mu \mathrm{m}$. The surface of the pores in the plate can be treated or coated to become able to produce photo and secondary electrons, and an electric field is placed over the two sides of the plate. A photon that is absorbed by a wall inside one of the pores may generate a free electron through the photoelectric effect. This electron is then accelerated by the electric field towards the anode. When it hits the wall of the pore again, it may generate several secondary electrons, resulting in an amplified cascade of electrons. The resulting charge pulse can be measured to determine the energy of the photon and the position where it was absorbed on the plate, resulting in a microchannel plate detector that can be used to measure energy and position of incoming visible to gamma ray photons and particles such as electrons, protons, neutrons and ions. The electron pulse can also be converted into several photons using a fluorescent screen, resulting in an image intensifier as for example used in night vision equipment.

The walls of these pores will also reflect X-rays under grazing incidence and can be used to focus $\mathrm{X}$-rays into a spot. However, because the original direction of the photons is lost upon one or more reflections on the round walls, it cannot be used to produce an imaging optics. This problem is overcome by using square fibres, which results in pores with flat, aligned walls. These walls can be used as reflecting surfaces to produce an imaging optics. Because of the extreme extension of the glass in the fibre production process, the roughness of the interface between square and cladding glass is reduced from that of a typical optical polish to the few $\mathrm{nm}$ roughness required for X-ray reflection.

The fibres can be placed in different geometries, each resulting in a different type of optic. For example, when the square fibres are placed in a circular geometry, the pore walls will reflect the X-rays from a point source towards a single focus. When the plate is also curved to a spherical shape, this will also happen for a source at a large distance.

However, proper imaging requires two reflections. This occurs with some of the X-rays that are reflected by a square array of fibres, namely, those rays that reflect from two adjacent orthogonal walls (i.e., an X-ray is reflected on one surface of the pore, and then hits the perpendicular surface of the adjacent wall of the same pore, where it is once more reflected). This results in a cross-shaped focus. When the plate is also curved to a spherical shape, the geometry resembles that of the eye of a lobster and images over a large field of view [38-44]. This technology can even be used to mimic the two surfaces of revolution that constitute a Wolter-I geometry for X-ray imaging $[45,46]$. This requires two plates, each with the fibres aligned in circles around the optical axis. The plates are curved to a spherical shape, where the radius of curvature of the second plate is $1 / 3$ of that of the first plate, and the plates are placed directly behind each other. X-rays from a source at a large distance, and that are reflected by the first and second plate consecutively, are imaged to a proper focus at a focal distance that is $1 / 4$ of the radius of curvature of the first plate. This also holds for 


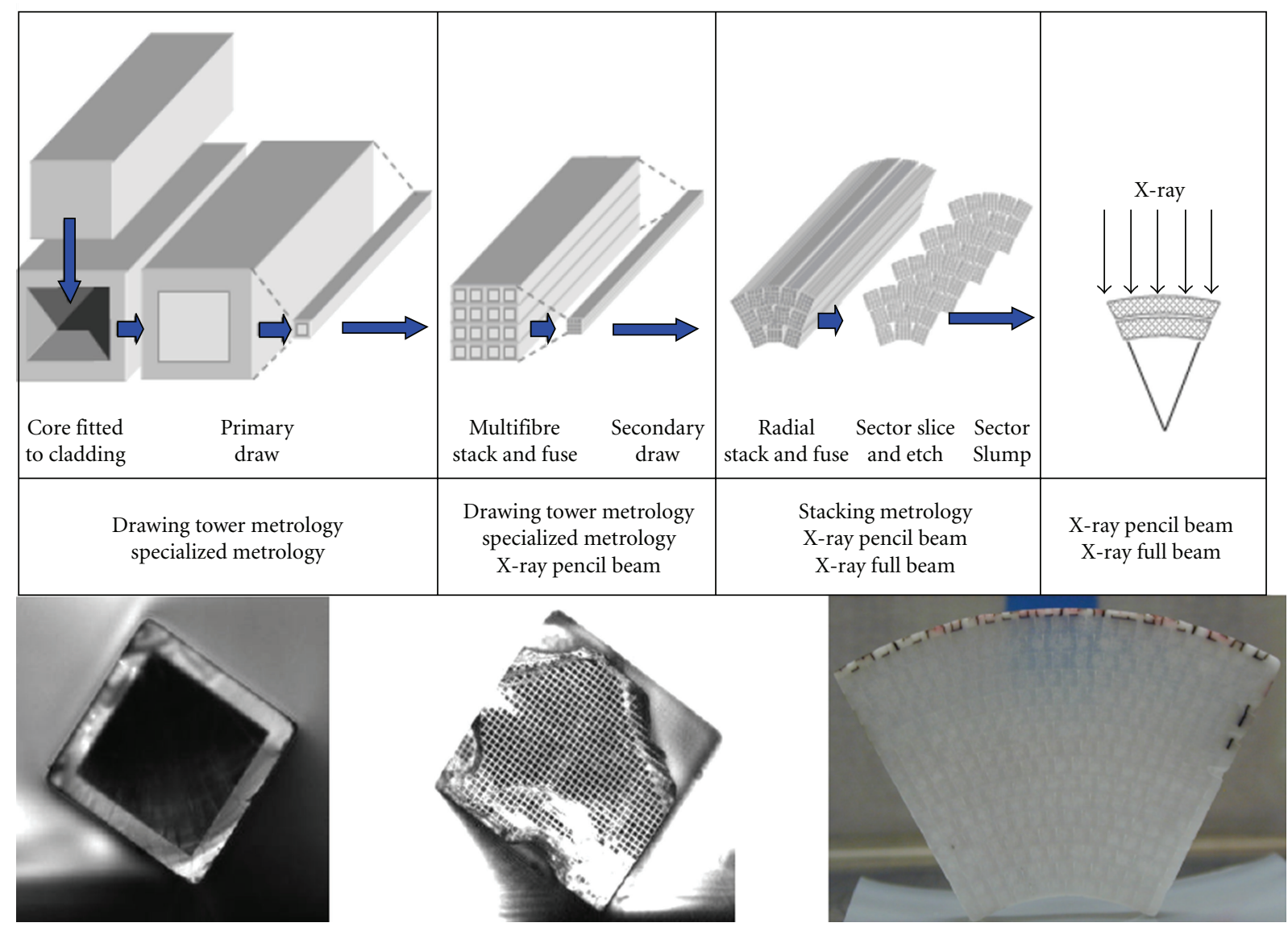

FIgURE 17: Manufacturing steps for micropore optics, reproduced from [47].

sources that are not on the optical axis, and therefore the two plates together form the equivalent of an X-ray "lens".

Larger MPOs can be tiled using MPO units, which themselves are limited in size (typically to less than $100 \mathrm{~mm}$ diameter) due to the manufacturing process. The angular resolution is currently limited to a few arc minutes.

3.2. Production of MPOs. The production of MPOs is derived from well established manufacturing methods of microchannel plates using drawing and stacking of glass fibres. But while microchannel plates contain cylindrical holes, X-ray optics need flat surfaces and consequently pores with a square profile. The required manufacturing steps are illustrated in Figure 17.

The base material is a polished glass block with a square profile that is surrounded with a cladding of a different glass type. Note that while the outer cladding forms the pore walls, its surface quality is mainly determined by the core glass block. A primary draw is performed in a draw tower, where the glass block is partially melted and drawn into a fibre by a traction system. The fibre is broken in regular pieces and stored. The geometry of the fibres is measured by dedicated optical metrology devices [47] during the drawing. Starting with a block size of approximately $20 \times 20 \times 300 \mathrm{~mm}^{3}$, the resulting fibres have a size of about $1 \times 1 \times 40 \mathrm{~mm}^{3}$.
A second drawing step is required to reach pore sizes in the order of a few $10 \mu \mathrm{m}$. Therefore, about $35 \times 35$ fibres are fused together, forming a multifibre stack. The second drawing results in multifibres with a pore size of $20 \times 20 \mu \mathrm{m}$ and a wall thickness of $3 \mu \mathrm{m}$. These multifibres are the basic building blocks for larger X-ray pore optics.

The multifibre stacks are again stacked and fused to form $\mathrm{X}$-ray optics with larger apertures, including the possibility to form a geometry of radial segments. Micropore optics plates with the desired geometry can be sliced from this block. The glass fibre cores are removed by selective etching of the glass type. The glass fibre cladding material forms the walls of a large number of holes with a square profile. Due to the high number of fused pores, the resulting element is of high structural stability.

The shape of a Wolter optic is approximated by thermally slumping separate micropore optic plates to form approximations to the hyperbolic and parabolic elements. In order to increase the reflectivity, metallic coatings such as nickel or iridium can be applied using an electroless deposition method. Two plates placed in front of each other form a focusing, micropore, X-ray optics.

3.3. Performance of Micropore Optics. The optical performance of the micropore optics strongly depends on the deformation of the individual fibres during the drawing, 

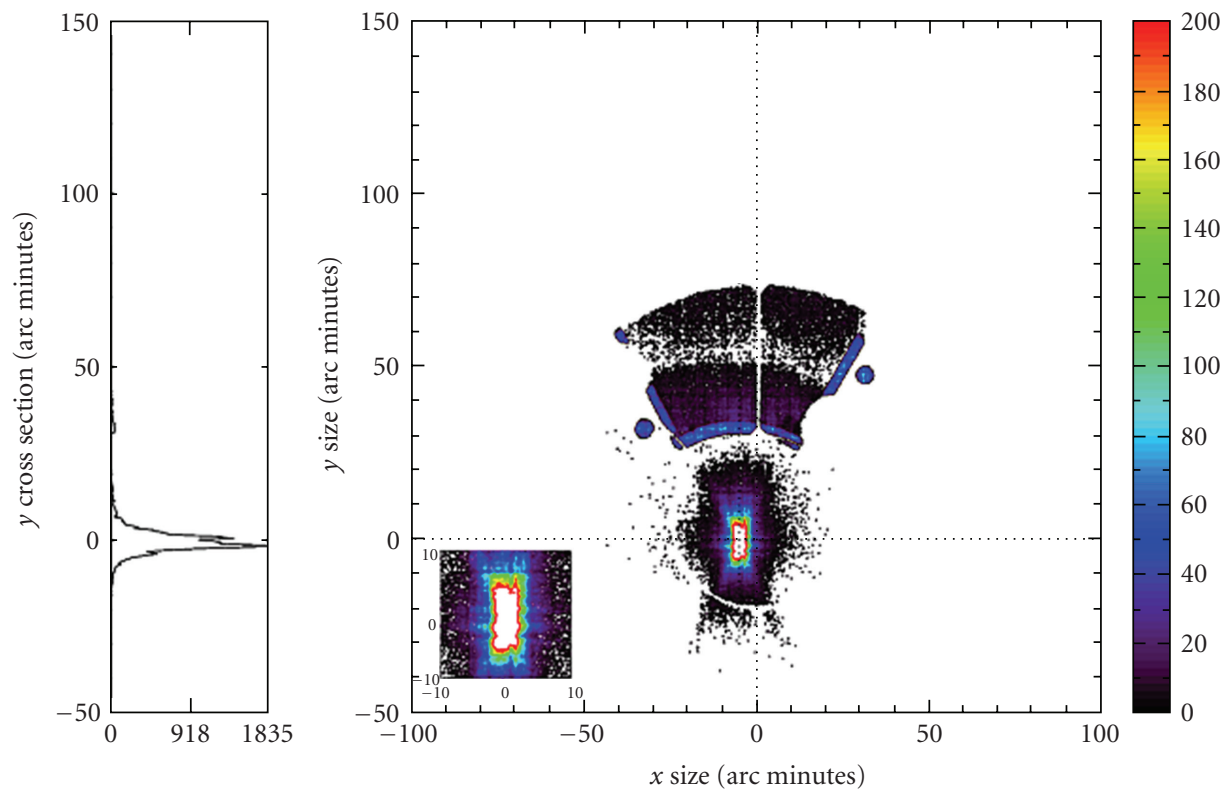

HEW 6.1 arcmin
FWHM-X 1.5 arcmin
FWHM-Y 3.9 arcmin

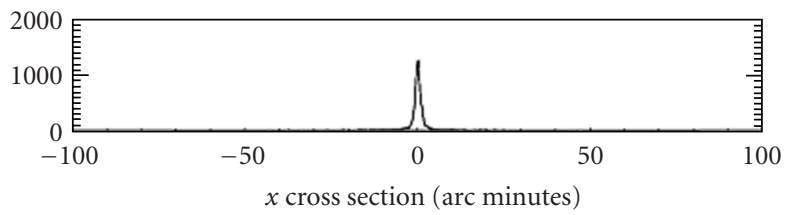

FIGURE 18: Full beam illumination measurement of a slumped sector, measured at $1.48 \mathrm{keV}$. The focus was found at the expected distance of $2 \mathrm{~m}$. The measured HEW of the focal spot is $6.1 \mathrm{arcmin}$. Even reflections and openings in the sample holder generate an image of the radial sector on the detector (visible above the focal spot).

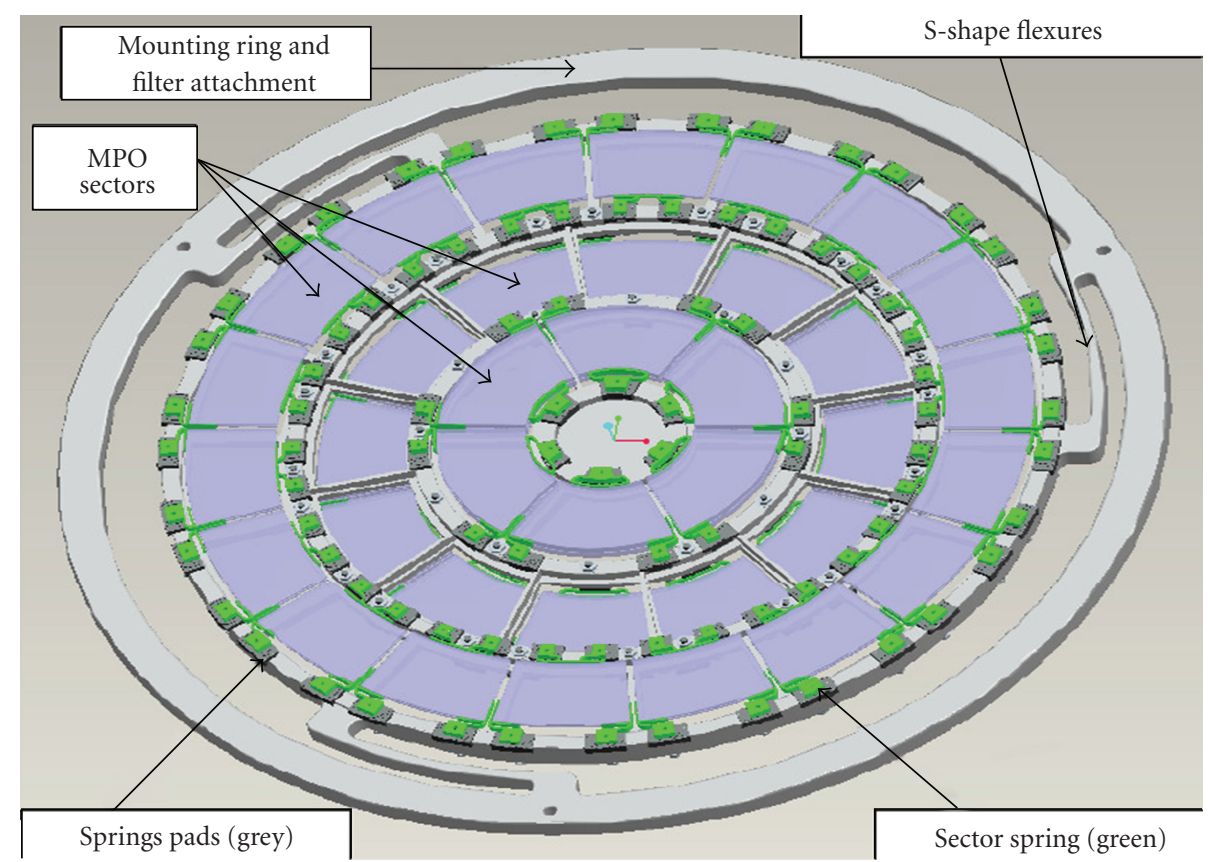

FIGURE 19: An early concept for assembling MPO sectors into an X-ray optics unit. The support structure is made of Titanium and must provide the correct and stable alignment of the sectors, while protecting them from thermal and mechanical loads. Three s-shaped flexures at the optic mount absorb mechanical loads, which would otherwise pass directly to the optic from the optical bench; they also provide a possible mount point for a filter. MPO sectors can be individually removed and replaced by unscrewing the bolts to the pads. The diameter of the optics is $200 \mathrm{~mm}$. 

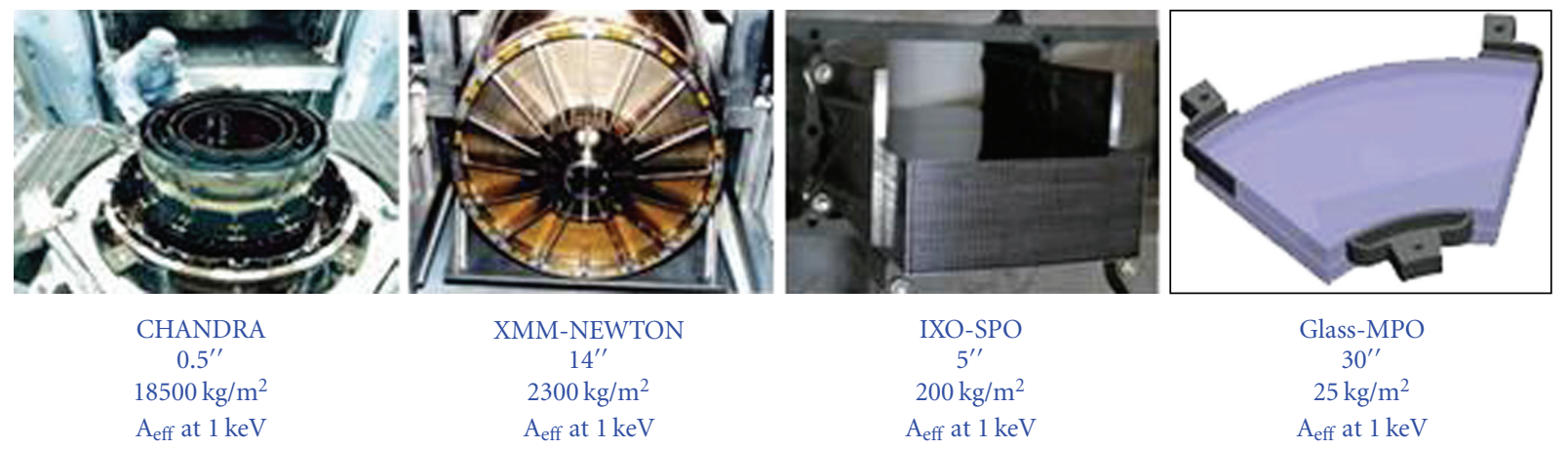

FIGURE 20: Comparison of X-ray optics technologies.

stacking and slumping processes. In order to understand and improve the production process, $\mathrm{X}$-ray measurements of the optics are performed after the different manufacturing steps [46-48]. Pencil beam measurements performed at the BESSY II beamlines of the Physikalisch-Technische Bundesanstalt (PTB) in Berlin are used for characterizing local properties of the micropore optics, using a $50 \mu \mathrm{m}$ beam size. Full beam illumination is done at the PANTER facility of the MPE in Munich.

Figure 18 shows a full beam illumination measurement from the first generation of slumped micropore optics sectors, about $50 \times 30 \mathrm{~mm}^{2}$ in size. At the design focal distance of $2 \mathrm{~m}$, the focal spot with a HEW of $6.1 \mathrm{arcmin}$ was measured. The focal position demonstrates the successful slumping of the $2.5 \mathrm{~mm}$ thick plate to a radius of curvature of $4 \mathrm{~m}$. The HEW of $6.1 \mathrm{arcmin}$ indicates that not all multifibres have been slumped correctly. Single reflection measurements on single multifibres have a typical HEW of 120 arcsec, where individual fibres within the multifibre are as good as 30 arc seconds [47], demonstrating the potential of the technology. In Figure 19, an early design is shown for assembling MPO sectors into complete optics.

An early concept for mounting of MPOs into an optics assembly is shown in Figure 19 [49].

3.4. Bepi-Colombo. In 2007, the MPO technology was chosen for implementation in the Mercury Imaging X-ray Spectrometer (MIXS). MIXS is an instrument of the Mercury Planetary Orbiter of the Bepi Colombo Mission [50, 51] planned to launch in 2014. The imaging spectrometer will measure X-ray fluorescence from Mercury within an energy range of $0.5-7.5 \mathrm{keV}$. It consists of two instruments [52]: one collimator (MIXS-C) and one telescope (MIXS-T) which uses a conical approximation of the Wolter-I configuration. The latter has a diameter of $210 \mathrm{~mm}$ and a focal length of $1 \mathrm{~m}$ and is required to yield a field of view of 1 degree with an angular resolution below 9 arcmin.

\section{Summary}

Pore Optics technology allows the production of high performance X-ray optics. The mass density required to achieve a given angular resolution is much lower than the established X-ray optics technologies. In Figure 20, the established optics technologies currently operating in orbit, on board Chandra and Newton, are compared with the SPO and the MPO technologies.

SPO technology has already demonstrated the required mass density for use on IXO and has shown very promising performance measured at X-ray facilities. A technology programme is being implemented, addressing further improvement of the angular resolution to reach the requirement of IXO, the predevelopment of mass production equipment necessary for large telescope implementations and, finally, the environmental qualification of SPO modules [35, 53-56].

MPO technology has achieved a maturity level adequate for adoption into a flight programme. This technology is the lightest, albeit with reduced angular resolution performance, and is suitable to meet the reduced mass and envelope requirements for planetary mission instrumentation.

The transfer of these technologies to ground applications is possible and attractive, in a wide range of fields, ranging from material science to medical diagnostics and security equipment.

\section{Acknowledgments}

The authors would like to acknowledge the work done by the industrial and institutional entities and the people involved in the technology preparation activities. Their work forms the basis onto which the future science missions are built. Without their efforts, the next generation X-ray astrophysics observatory, IXO, could not be realised, and planetary X-ray optics would not be possible. The MPO section of this paper is dedicated to the memory of Sven-Olof Flyckt. His genuine kindness, his passion, and his expertise were not only vital for the technical but also for the human aspects of such projects.

\section{References}

[1] F. Jansen, D. Lumb, B. Altieri et al., "XMM-Newton observatory. I. The spacecraft and operations," Astronomy and Astrophysics, vol. 365, no. 1, pp. L1-L6, 2001.

[2] S. L. O'Dell and M. C. Weisskopf, "Advanced X-ray astrophysics facility (AXAF):calibration overview," in X-Ray Optics, Instruments, and Missions, vol. 3444 of Proceedings of SPIE, pp. 2-18, San Diego, Calif, USA, July 1998. 
[3] M. J. L. Turner, G. G. C. Palumbo, J. A. M. Bleeker, G. Hasinger, A. Peacock, and J. Trumper, "Xeus: an X-ray observatory for the post-XMM era," in The Next Generation of X-Ray Observatories: Workshop Proceedings Leicester X-ray Astronomy Group Special Report XRA 97/02, M. J. L. Turner and M. G. Watson, Eds., p. 165, 1997.

[4] A. Parmar, G. Hasinger, and M. Turner, "XEUS - the X-ray evolving universe spectroscopy mission," in Proceedings of the 34th Cospar Assembly, p. 2368, 2004.

[5] G. Bignami et al., "Cosmic vision,” ESA BR-247, 2005.

[6] B. Aschenbach, "X-ray telescopes," Reports on Progress in Physics, vol. 48, no. 5, pp. 579-629, 1985.

[7] A. Peacock, B. G. Taylor, and J. Ellwood, "The high throughput X-ray spectroscopy mission: XMM," Advances in Space Research, vol. 10, no. 2, pp. 273-285, 1990.

[8] P. Gondoin, B. R. Aschenbach, H. Brauninger et al., "X-ray performance of a qualification model of an XMM mirror module," in EUV, X-Ray, and Gamma-Ray Instrumentation for Astronomy VII, vol. 2808 of Proceedings of SPIE, pp. 390-401, Denver, Colo, USA, August 1996.

[9] M. Bavdaz, J. A. M. Bleeker, G. Hasinger et al., "X-ray evolving universe spectroscopy mission (XEUS): narrow-field imaging high-resolution spectrometer: II (1 to $10 \mathrm{keV})$," in X-Ray Optics, Instruments, and Missions II, vol. 3766 of Proceedings of SPIE, pp. 82-93, Denver, Colo, USA, July 1999.

[10] M. Bavdaz, A. J. Peacock, A. N. Parmar, M. Beijersbergen, and J. Schiemann, "The X-ray evolving universe spectroscopy mission (XEUS)," in The Role of the International Space Station. ESA SP-433: Utilisation of the International Space Station 2, p. 621, 1999.

[11] M. Bavdaz, M. Beijersbergen, A. J. Peacock, R. Willingale, B. R. Aschenbach, and H. W. Braeuninger, "X-ray evolving universe spectroscopy mission (XEUS): X-ray mirror design and technology," in X-Ray Optics, Instruments, and Missions II, R. B. Hoover and A. B. Walker, Eds., vol. 3766 of Proceedings of SPIE, pp. 94-102, Denver, Colo, USA, July 1999.

[12] W. W. Zhang, K.-W. Chan, T. Hajimichael et al., "Development of lightweight X-ray mirrors for the constellationX mission," in Optics for EUV, X-Ray, and Gamma-Ray Astronomy III, vol. 6688 of Proceedings of SPIE, San Diego, Calif, USA, August 2007.

[13] M. Bavdaz, A. Peacock, E. Tomaselli et al., "Progress at ESA on high energy optics technologies," in Optics for EUV, X-Ray, and Gamma-Ray Astronomy, vol. 5168 of Proceedings of SPIE, pp. 136-147, San Diego, Calif, USA, August 2003.

[14] M. Beijersbergen, S. Kraft, M. Bavdaz et al., "Development of X-ray pore optics: novel high-resolution silicon millipore optics for XEUS and ultra-low mass glass micropore optics for imaging and timing," in Design and Microfabrication of Novel X-Ray Optics II, vol. 5539 of Proceedings of SPIE, pp. 104-115, Denver, Colo, USA, August 2004.

[15] P. Friedrich, B. Aschenbach, C. Braig et al., "Manufacturing of Wolter-I mirror segments with slumped glass," in Space Telescopes and Instrumentation II: Ultraviolet to Gamma Ray, vol. 6266 of Proceedings of SPIE, Orlando, Fla, USA, May 2006.

[16] M. Ghigo, O. Citterio, F. Mazzoleni et al., "The manufacturing of the XEUS X-ray glass segmented mirrors: status of the investigation and last results," in Optics for EUV, X-Ray, and Gamma-Ray Astronomy, vol. 5168, pp. 180-195, San Diego, Calif, USA, August 2003.

[17] M. Beijersbergen, S. Kraft, R. Günther et al., "Silicon pore optics: novel lightweight high-resolution X-ray optics developed for XEUS," in UV and Gamma-Ray Space Telescope
Systems, vol. 5488 of Proceedings of SPIE, pp. 868-874, Glasgow, UK, June 2004.

[18] S. Kraft, M. Collon, R. Guenther et al., "Development of modular High-performance pore optics for the XEUS Xray telescope," in Optics for EUV, X-Ray, and Gamma-Ray Astronomy II, vol. 5900 of Proceedings of SPIE, pp. 297-308, San Diego, Calif, USA, August 2005.

[19] M. J. Collon, S. Kraff, R. Günther et al., "Metrology, integration and performance verification of silicon pore optics in Wolter-I configuration," in Space Telescopes and Instrumentation II: Ultraviolet to Gamma Ray, vol. 6266 of Proceedings of SPIE, Orlando, Fla, USA, May 2006.

[20] Q. Wang, U. Griesmann, and R. Polvani, "Interferometric thickness calibration of $300 \mathrm{~mm}$ silicon wafers," in ASPE Summer Topical Meeting on Precision Interferometric Metrology, July 2005.

[21] M. B. O. Riekerink, B. Lansdorp, L. J. de Vreede et al., "Production of silicon mirror plates," in Optics for EUV, XRay, and Gamma-Ray Astronomy IV, vol. 7437 of Proceedings of SPIE, p. 27, San Diego, Calif, USA, August 2009.

[22] M. D. Ackermann, M. J. Collon, R. Günther et al., "Performance prediction and measurement of silicon pore optics," in Optics for EUV, X-Ray, and Gamma-Ray Astronomy IV, vol. 7437 of Proceedings of SPIE, San Diego, Calif, USA, August 2009.

[23] H. Wolter, "Spiegelsysteme streifenden Einfalls als abbildende Optiken fur Rontgenstrahlen," Annalen der Physik, vol. 445, pp. 94-114, 1952.

[24] D. H. Lumb, F. E. Christensen, C. P. Jensen, and M. Krumrey, "Influence of a carbon over-coat on the X-ray reflectance of XEUS mirrors," Optics Communications, vol. 279, no. 1, pp. 101-105, 2007.

[25] C. P. Jensen, M. Ackermann, F. E. Christensen, M. J. Collon, and M. Krumrey, "Coating of silicon pore optics," in Optics for EUV, X-Ray, and Gamma-Ray Astronomy IV, vol. 7437 of Proceedings of SPIE, San Diego, Calif, USA, August 2009.

[26] Q.-Y. Tong and U. Gösele, Semiconductor Wafer Bonding: Science and Technology, John Wiley \& Sons, New York, NY, USA, 1999.

[27] A. L. Mieremet and M. W. Beijersbergen, "Fundamental spatial resolution of an X-ray pore optic," Applied Optics, vol. 44, no. 33, pp. 7098-7105, 2005.

[28] R. Günther, M. Collon, S. Kraft et al., "Production of silicon pore optics," in Space Telescopes and Instrumentation II: Ultraviolet to Gamma Ray, vol. 6266 of Proceedings of SPIE, Orlando, Fla, USA, May 2006.

[29] M. J. Collon, R. Günther, S. Kraft et al., "Silicon pore optics for astrophysical X-ray missions," in Optics for EUV, X-Ray, and Gamma-Ray Astronomy III, vol. 6688 of Proceedings of SPIE, San Diego, Calif, USA, August 2007.

[30] M. J. Collon, S. Kraft, R. Günther et al., "Performance characterization of silicon pore optics," in Space Telescopes and Instrumentation II: Ultraviolet to Gamma Ray, vol. 6266 of Proceedings of SPIE, Orlando, Fla, USA, May 2006.

[31] M. Krumrey and G. Ulm, "High-accuracy detector calibration at the PTB four-crystal monochromator beamline," Nuclear Instruments and Methods in Physics Research, Section A, vol. 467-468, pp. 1175-1178, 2001.

[32] R. Graue, D. Kampf, S. Kraft et al., "Assembling silicon pore optics into a modular structure," in Space Telescopes and Instrumentation II: Ultraviolet to Gamma Ray, vol. 6266 of Proceedings of SPIE, Orlando, Fla, USA, May 2006. 
[33] M. Freyberg, B. Budau, W. Burkert et al., "Potential of the PANTER X-ray test facility for calibration of instrumentation for XEUS," in Space Telescopes and Instrumentation II: Ultraviolet to Gamma Ray, vol. 6266 of Proceedings of SPIE, Orlando, Fla, USA, May 2006.

[34] E. J. Buis and G. Vacanti, "X-ray tracing using Geant4," Nuclear Instruments and Methods in Physics Research, Section A, vol. 599, no. 2-3, pp. 260-263, 2009.

[35] M. Bavdaz, PH. Gondoin, K. Wallace et al., "IXO system studies and technology preparation," in Optics for EUV, XRay, and Gamma-Ray Astronomy IV, vol. 7437 of Proceedings of SPIE, San Diego, Calif, USA, August 2009.

[36] D. Martin, N. Rando, D. Lumb et al., "Payload study activities on the international X-ray observatory," in Space Telescopes and Instrumentation: Ultraviolet to Gamma Ray, vol. 7732 of Proceedings of SPIE, San Diego, Calif, USA, June 2010.

[37] Y. Ezoe, M. Koshiishi, M. Mita et al., "Micropore X-ray optics using anisotropic wet etching of (110) silicon wafers," Applied Optics, vol. 45, no. 35, pp. 8932-8938, 2006.

[38] W. K. H. Schmidt, "A proposed X-ray focusing device with wide field of view for use in X-ray astronomy," Nuclear Instruments and Methods, vol. 127, no. 2, pp. 285-292, 1975.

[39] J. R. P. Angel, "Lobster eyes as X-ray telescopes," Astrophysical Journal, vol. 233, pp. 364-373, 1979.

[40] S. W. Wilkins, A. W. Stevenson, K. A. Nugent, H. Chapman, and S. Steenstrup, "On the concentration, focusing, and collimation of X-rays and neutrons using microchannel plates and configurations of holes," Review of Scientific Instruments, vol. 60, no. 6, pp. 1026-1036, 1989.

[41] G. W. Fraser, J. E. Lees, J. F. Pearson, M. R. Sims, and K. Roxburgh, "X-ray focusing using microchannel plates," in Multilayer and Grazing Incidence X-Ray/EUV Optics, R. B. Hoover, Ed., vol. 1546 of Proceedings of SPIE, pp. 41-52, San Diego, Calif, USA, 1992.

[42] G. W. Fraser, A. N. Brunton, J. E. Lees, and D. L. Emberson, "Production of quasi-parallel X-ray beams using microchannel plate "X-ray lenses"," Nuclear Instruments and Methods in Physics Research A, vol. 334, no. 2-3, pp. 579-588, 1993.

[43] A. G. Peele, B. E. Allman, and V. Cucevic, "Square capillary Xray optics," in Applications of Laser Plasma Radiation, vol. 2015 of Proceedings of SPIE, pp. 118-127, San Diego, Calif, USA, July 1993.

[44] A. N. Brunton, G. W. Fraser, J. E. Lees, and I. C. E. Turcu, "Metrology and modeling of microchannel plate X-ray optics," Applied Optics, vol. 36, no. 22, pp. 5461-5470, 1997.

[45] R. Willingale, G. W. Fraser, A. N. Brunton, and A. P. Martin, "Hard X-ray imaging with microchannel plate optics," Experimental Astronomy, vol. 8, no. 4, pp. 281-296, 1998.

[46] M. Beijersbergen, M. Bavdaz, A. Peacock et al., "Microchannel plate based X-ray optics," in EUV, X-Ray, and Gamma-Ray Instrumentation for Astronomy X, vol. 3765 of Proceedings of SPIE, pp. 452-459, Denver, Colo, USA, July 1999.

[47] M. J. Collon, M. W. Beijersbergen, K. Wallace et al., "X-ray imaging glass micro-pore optics," in Optics for EUV, X-Ray, and Gamma-Ray Astronomy III, vol. 6688 of Proceedings of SPIE, San Diego, Calif, USA, August 2007.

[48] K. Wallace, M. Collon, M. Bavdaz, R. Fairbend, J. Séguy, and M. Krumrey, "Developments in glass micro pore optics for Xray applications," in Space Telescopes and Instrumentation II: Ultraviolet to Gamma Ray, vol. 6266 of Proceedings of SPIE, p. 37, Orlando, Fla, USA, May 2006.
[49] K. Wallace, M. J. Collon, M. W. Beijersbergen, S. Oemrawsingh, M. Bavdaz, and E. Schyns, "Breadboard micro-pore optic development for X-ray imaging," in Optics for EUV, XRay, and Gamma-Ray Astronomy III, vol. 6688 of Proceedings of SPIE, p. 44, San Diego, Calif, USA, August 2007.

[50] A. Owens, M. Bavdaz, M. Beijersbergen et al., "HERMES: an imaging X-ray fluorescence spectrometer for the BepiColombo mission to Mercury," in Soft X-Ray and EUV Imaging Systems II, vol. 4506 of Proceedings of SPIE, pp. 136145, San Diego, Calif, USA, August 2001.

[51] M. Collon, E. J. Buis, M. Beijersbergen et al., "Design and performance of the payload instrumentation of the BepiColombo Mercury planetary orbiter," Acta Astronautica, vol. 59, no. 8-11, pp. 1052-1061, 2006.

[52] G. W. Fraser, J. D. Carpenter, D. A. Rothery et al., "The mercury imaging X-ray spectrometer (MIXS) on bepicolombo," Planetary and Space Science, vol. 58, no. 1-2, pp. 79-95, 2009.

[53] M. D. Ackermann, M. J. Collon, C. P. Jensen et al., "Performance of multilayer coated silicon pore optics," in Space Telescopes and Instrumentation: Ultraviolet to Gamma Ray, vol. 7732 of Proceedings of SPIE, San Diego, Calif, USA, June 2010.

[54] M. Bavdaz, E. Wille, K. Wallace et al., "ESA optics technology preparation for IXO," in Space Telescopes and Instrumentation: Ultraviolet to Gamma Ray, vol. 7732 of Proceedings of SPIE, San Diego, Calif, USA, June 2010.

[55] M. J. Collon, R. Günther, M. Ackermann et al., "Stacking of silicon pore optics for IXO," in Optics for EUV, X-Ray, and Gamma-Ray Astronomy IV, vol. 7437 of Proceedings of SPIE, San Diego, Calif, USA, August 2009.

[56] N. Rando, D. Martin, D. Lumb et al., "ESA assessment study activities on the International X-ray observatory," in Space Telescopes and Instrumentation: Ultraviolet to Gamma Ray, vol. 7732 of Proceedings of SPIE, San Diego, Calif, USA, June 2010. 